\title{
The 3 Ms Chandra campaign on Sgr A*: a census of X-ray flaring activity from the Galactic center
}

\author{
J. Neilsen ${ }^{1,2}$, M. A. Nowak ${ }^{2}$, C. Gammie ${ }^{3}$, J. Dexter ${ }^{4}$, S. Markoff ${ }^{5}$,
}

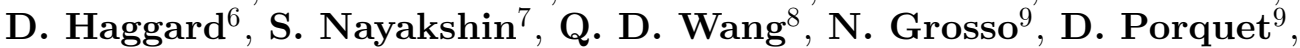
J. A. Tomsick ${ }^{10}$, N. Degenaar ${ }^{11}$, P. C. Fragile ${ }^{12}$, J. C. Houck ${ }^{2}$, R. Wijnands ${ }^{5}$, J. M. Miller ${ }^{11}$ and F. K. Baganoff ${ }^{2}$

${ }^{1}$ Einstein Fellow, Boston University, 725 Commonwealth Ave, Boston MA 02215 email: neilsenj@bu.edu

${ }^{2}$ MIT Kavli Institute for Astrophysics and Space Research, Cambridge MA 02139

${ }^{3}$ Departments of Astronomy \& Physics, Univ. of Illinois Urbana-Champaign, Urbana IL 61801

${ }^{4}$ Theoretical Astrophysics Center and Department of Astronomy, UC Berkeley, CA 94720-3411

${ }^{5}$ Astronomical Institute "Anton Pannekoek", Univ. Amsterdam, 1098 XH Amsterdam, NL

${ }^{6}$ CIERA Fellow, Physics and Astronomy Department, Northwestern Univ., Evanston, IL 60208

${ }^{7}$ Department of Physics \& Astronomy, University of Leicester, Leicester LE1 7RH UK

${ }^{8}$ Department of Astronomy, University of Massachusetts, Amherst, MA 01002

${ }^{9}$ Observatoire Astronomique de Strasbourg, Université de Strasbourg, CNRS, 67000 Strasbourg, FR

${ }^{10}$ Space Sciences Laboratory, 7 Gauss Way, University of California, Berkeley, CA 94720

${ }^{11}$ Department of Astronomy, University of Michigan, Ann Arbor, MI 48109; ND: Hubble Fellow

${ }^{12}$ Department of Physics \& Astronomy, College of Charleston, Charleston, SC 29424

\begin{abstract}
Over the last decade, X-ray observations of Sgr A* have revealed a black hole in a deep sleep, punctuated roughly once per day by brief flares. The extreme X-ray faintness of this supermassive black hole has been a long-standing puzzle in black hole accretion. To study the accretion processes in the Galactic center, Chandra (in concert with numerous ground- and space-based observatories) undertook a $3 \mathrm{Ms}$ campaign on Sgr A* in 2012. With its excellent observing cadence, sensitivity, and spectral resolution, this Chandra X-ray Visionary Project (XVP) provides an unprecedented opportunity to study the behavior of the closest supermassive black hole. We present a progress report from our ongoing study of X-ray flares, including the brightest flare ever seen from Sgr A*. Focusing on the statistics of the flares and the quiescent emission, we discuss the physical implications of X-ray variability in the Galactic center.
\end{abstract}

Keywords. accretion, accretion disks, black hole physics, radiation mechanisms: nonthermal

\section{Introduction}

In the last fifteen years, X-ray observations of $\operatorname{Sgr~A}^{*}$, the $4 \times 10^{6} \mathrm{M}_{\odot}$ black hole at the center of our Galaxy, have revealed a profoundly quiescent supermassive black hole, its inactivity punctuated roughly once a day by rapid flares (e.g. Baganoff et al. 2001, 2003; Dodds-Eden et al. 2009; Genzel et al. 2010; Markoff 2010 and references therein). Short flares are clearly observed from the sub-mm (Marrone et al. 2008) to the hard Xray (Barriere et al. 2013). The flares make a particularly appealing target because, given the paucity of thorough multi-wavelength coverage, it has historically been difficult to constrain either the flare mechanism or the dominant radiation process (e.g., synchrotron, synchrotron self-Compton, external Compton).

But despite surpassing the quiescent luminosity of Sgr A* by factors of 100 or more in the X-ray band (e.g. Porquet et al. 2003, 2008; Nowak et al. 2012), the flares have 


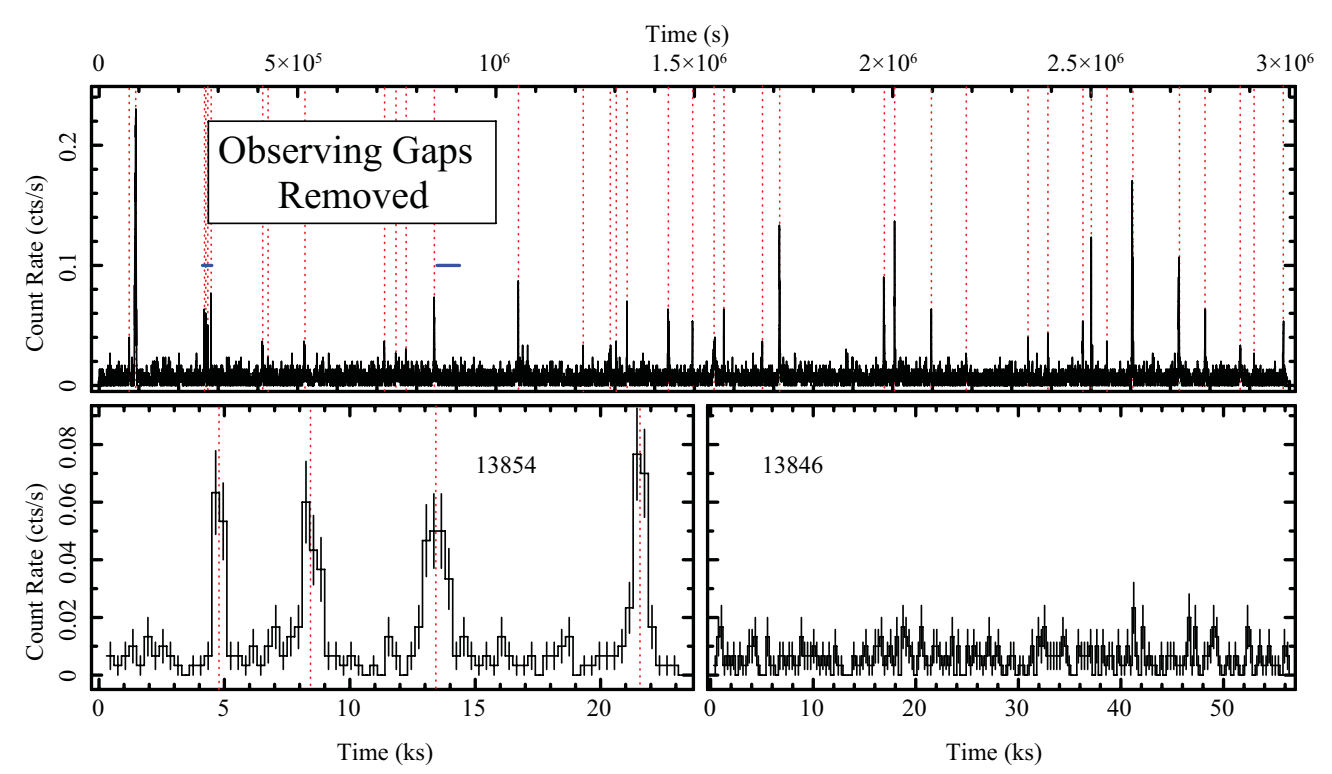

Figure 1. From Neilsen et al. (2013). (c) 2013. The American Astronomical Society. All rights reserved.) (Top): $2-8 \mathrm{keV}$ Chandra X-ray lightcurve of $\mathrm{Sgr} \mathrm{A}^{*}$ in $300 \mathrm{~s}$ bins for the entire 2012 XVP campaign with gaps removed. Numerous flares at a range of intensities are apparent, and are identified by dotted red lines. Horizontal lines mark sample observations shown in the bottom plots. (Bottom): Sample light curves of an observation with (left) and without (right) detected flares. ObsID 13854 (left) shows four moderately bright flares within $20 \mathrm{ks}$. If the flare rate is taken as a constant during 2012 , the probability of such a cluster is $\lesssim 3.5 \%$. [A COLOR VERSion IS AVALLAbLE ONLINE.]

actually deepened the puzzle of the ultra-low luminosity of our closest supermassive black hole. Across the mass scale, most weakly accreting black holes fall on the Fundamental Plane of black hole activity (FP), a three-way correlation between black hole mass, X-ray luminosity, and radio luminosity (e.g., Merloni et al. 2003; Falcke et al. 2004). Sgr A* is a notable exception, approaching the FP only during its daily flares (Markoff 2005; Plotkin et al. 2012). These flares therefore trace a link between Sgr A* and other black holes at very low accretion rates, although these connections cannot explain why Sgr A* does not always fall on the FP.

The 2012 Chandra X-ray Visionary Project (XVP) provides a prime opportunity to study the physics of X-ray flares and their relationship to the quiescent X-ray emission. This 3 Ms campaign to observe the Galactic center at the highest spatial and spectral resolution available in the X-ray band has the goals of (1) using high-resolution spectra to probe the physics of the accretion flow (see Xu et al. 2006; Young et al. 2007; Wang et al. 2013) and (2) understanding the origin and significance of flares from Sgr A*. Here, we provide a progress report on our analysis of flares detected during the XVP. Details can be found in Neilsen et al. (2013).

\section{Flare properties and statistics}

Between February and October of 2012, Chandra observed the Galactic center 38 times; the complete light curve of Sgr A* from the XVP campaign is shown (with observing gaps removed) in Figure 1. A number of bright flares are readily detectable above a steady background (constant within observational uncertainties, this background includes 

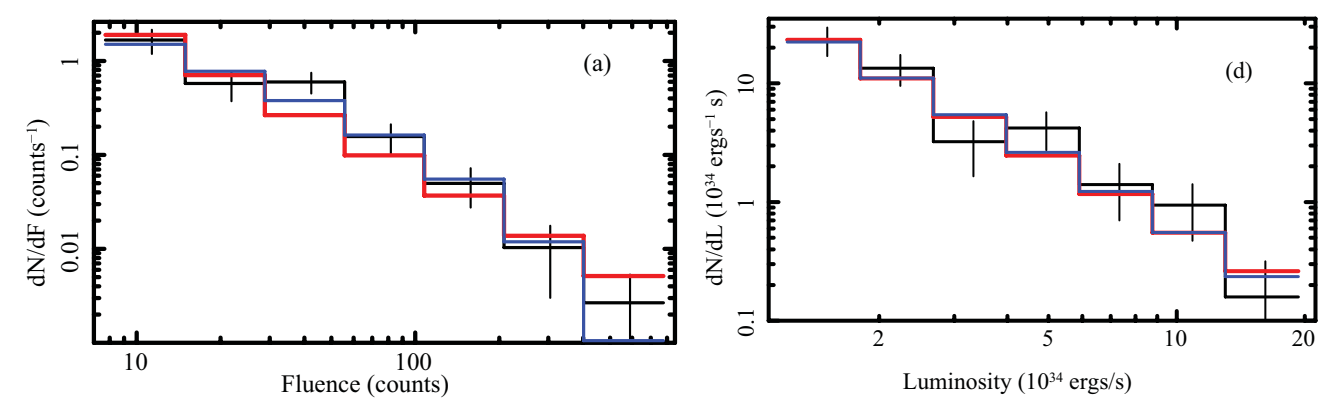

Figure 2. From Neilsen et al. (2013). (c) 2013. The American Astronomical Society. All rights reserved.) Distributions of flare fluence (right) and estimated mean unabsorbed 2-10 $\mathrm{keV}$ luminosity (left). The black histograms represent distributions corrected for photon pileup and incompleteness, with Poisson errors on the number of flares in each bin. The red curves are the best power law fit, and the blue curves are the best cutoff power law fit. [A COLOR VERSion is AVAILABLE ONLINE.]

emission from the diffuse gas in the Galactic center and from the quiescent accretion flow, e.g., Wang et al. 2013). To detect and characterize these flares, we fit the light curve of each observation with a model consisting of a constant and superimposed Gaussian flares (see Neilsen et al. 2013 for details). We identify 39 flares during the XVP, including the brightest X-ray flare ever observed from Sgr A* (peak luminosity $L_{\mathrm{X}} \sim 5 \times 10^{35} \mathrm{erg} \mathrm{s}^{-1}$; Nowak et al. 2012).

The average observed flare is somewhat more modest, lasting roughly $2600 \mathrm{~s}$ and peaking around 0.06 counts $\mathrm{s}^{-1}(2-8 \mathrm{keV})$. Scaling from the spectral analysis of the brightest flare, we can estimate a typical mean $2-10 \mathrm{keV}$ flare luminosity of $\sim 5 \times 10^{34} \mathrm{erg}$ $\mathrm{s}^{-1}$. The brightest flares seen by Chandra and $X M M$ have power law spectra with photon indices $\Gamma \sim 2$ (Nowak et al. 2012 and references therein), and we see no evidence for any luminosity-dependent variations in the flare hardness ratio (although see Degenaar et al. 2013; Barriere et al. 2013). While the analysis of the hardness ratios and spectra of individual flares has not yet conclusively ruled out any flare models, we can glean additional insights from the improved statistics afforded by the XVP.

In Figure 2, we show the completeness-corrected differential distributions of the fluence (total 2-8 keV counts) and estimated mean 2-10 keV luminosity of the flares. The luminosity distribution is consistent with a power law $d N / d L \propto L^{-1.9 \pm 0.4}$, which is similar to what is observed in solar flares (Crosby 2011, although the flares from Sgr A* are too bright to be stellar in origin) and to what may be expected from the tidal disruption of asteroids if the asteroid mass distribution in the Galactic center is comparable to that in the solar system (Zubovas et al. 2012). For other models such as shocks and reconnection (e.g., Markoff et al. 2001), it is still difficult (if not impossible) to predict luminosity distributions from first principles.

The fluence distribution scales like $d N / d F \sim F^{-1.5 \pm 0.2}$, so that the radiant energy is dominated by bright flares. Adding up the observed fluences, we find that $1 / 3$ of the total emitted energy during our $3 \mathrm{Ms}$ campaign was detected in flares, which have a duty cycle of roughly $\sim 3.5 \%$, so the typical flare is about $\sim 10 \times$ brighter than the background emission. In addition to counting up the observed flares, we can explore the contribution of undetected flares to the quiescent level. Integrating backwards, below our detection limit, we find that the fluence in undetected flares is $\lesssim 10 \%$ of the quiescent emission.

The contribution of weak/undetected flares to the quiescent emission can also be constrained via variability analysis. In the left panel of Figure 3, we show the observed distribution of waiting times between quiescent photons. For a pure Poisson process 

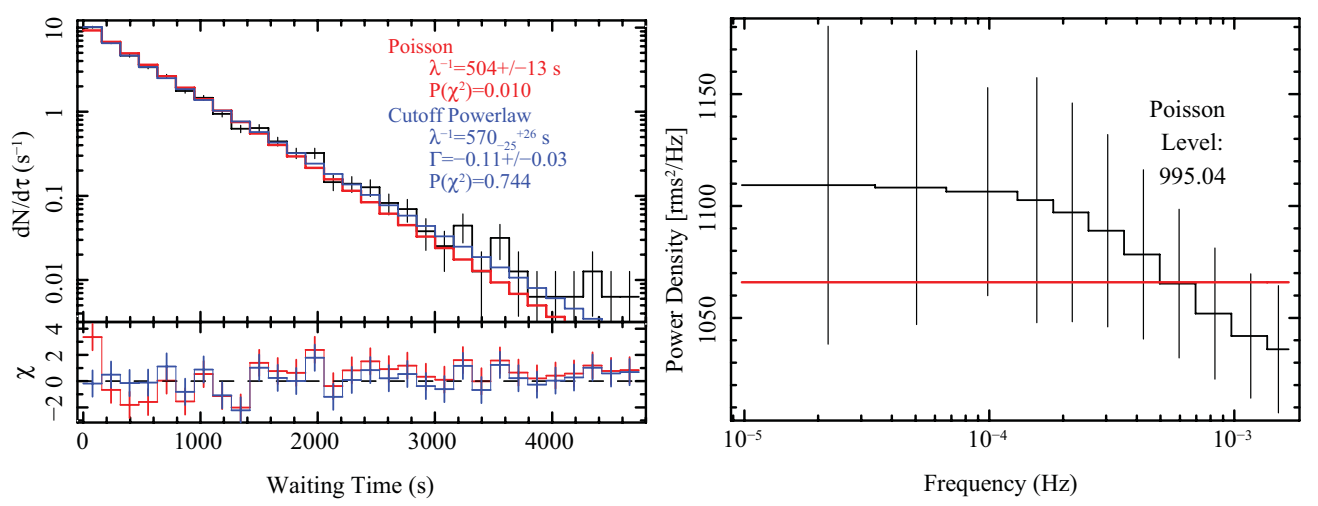

Figure 3. From Neilsen et al. (2013). (c) 2013. The American Astronomical Society. All rights reserved.) (Left): Distribution of times between quiescent photons, fit with an exponential model and a cutoff power law model (blue). (Right): The estimated power spectrum of the quiescent emission from $\mathrm{Sgr} \mathrm{A}$ * is statistically indistinguishable from white noise, and is shown with the best fit constant model. We find $\mathrm{a} \sim 10 \%$ excess above the pure Poisson noise level. [A COLOR VERsion is aVAilable online.]

(such as might be expected from thermal plasma on scales comparable to the Bondi radius; Wang et al. 2013), this distribution should be exponential, but we find evidence of an additional component of correlated variability. Monte Carlo estimates of the power density spectrum in quiescence indicate that this component is consistent with white noise, but provides an excess power of $\sim 10 \%$ over the Poisson level.

\section{Discussion: seen and unseen}

From the Chandra light curve in Figure 1, it is clear that the X-ray emission from Sgr A* comes in at least two flavors: one steady throughout 2012 and one rapidly variable on time scales as short as $100 \mathrm{~s}$ (Nowak et al. 2012). But a close inspection of the quiescent emission reveals that it is not completely steady: there is a $\sim 10 \%$ variability excess, which could be caused by undetected flares. This interpretation is consistent with a model in which the observed flare distribution extends to very low fluence and undetected flares contribute as much as $10 \%$ of the quiescent flux. Remarkably, spectral analysis of the quiescent emission (Wang et al. 2013) requires a nonthermal (power-law) component to explain $\sim 10-20 \%$ of the flux, and models of the surface brightness of Sgr A* require a point source to explain $10 \%$ of the flux (the rest is extended; Shcherbakov \& Baganoff 2010; Wang et al. 2013). We conclude that the flare statistics, along with the variability, spectrum, and surface brightness profile of Sgr A*, provide a consistent physical decomposition of its X-ray emission in quiescence: $\sim 90 \%$ is steady thermal plasma emission on scales comparable to the Bondi radius, and $\sim 10 \%$ is power-law emission from flares close to the event horizon. Future statistical studies comparing Xray and infrared variability (Dodds-Eden et al. 2011; Witzel et al. 2012; Neilsen et al., in prep) will provide deeper insight into the radiation physics of these exciting flares.

\section{References}

Baganoff, F. K., et al. 2001, Nature 413, 45

—. 2003, ApJ 591, 891

Barriere, N., et al. 2013, in AAS/High Energy Astrophysics Division, Vol.13, \#403.02

Crosby, N. B. 2011, Nonlinear Processes in Geophysics, 18, 791 
Degenaar, N., et al. 2013, ApJ 769, 155

Dodds-Eden, K., et al. 2011, ApJ 728, 37

-. 2009, ApJ 698, 676

Falcke, H., Körding, E., \& Markoff, S. 2004, A\&A 414, 895

Genzel, R., Eisenhauer, F., \& Gillessen, S. 2010, Reviews of Modern Physics, 82, 3121

Markoff, S. 2005, ApJ Lett. 618, L103

—. 2010, Proceedings of the National Academy of Science, 107, 7196

Markoff, S., et al. 2001, A\&A 379, L13

Marrone, D. P., et al. 2008, ApJ 682, 373

Merloni, A., Heinz, S., \& di Matteo, T. 2003, MNRAS 345, 1057

Neilsen, J., et al. 2013, ApJ 774, 42

Nowak, M. A., et al. 2012, ApJ 759, 95

Plotkin, R. M., et al. 2012, MNRAS 419, 267

Porquet, D., et al. 2008, A\&A 488, 549

-. 2003, A\& $\&$ 407, L17

Shcherbakov, R. V., \& Baganoff, F. K. 2010, ApJ 716, 504

Wang, Q. D., et al. 2013, Science, 341, 981

Witzel, G., et al. 2012, ApJS 203, 18

$\mathrm{Xu}, \mathrm{Y} .-\mathrm{D} .$, et al. 2006, ApJ 640, 319

Young, A. J., et al. 2007, ApJ 669, 830

Zubovas, K., Nayakshin, S., \& Markoff, S. 2012, MNRAS 421, 1315 